\title{
Herramienta GIS y servicios web en la geolocalización como instrumento en la adecuada gestión del territorio: Geoportal IDE Chinácota
}

Recibido:

Abril 25 de 2013

Aceptado:

Mayo 30 de 2013
${ }^{1} \mathrm{Ph} . \mathrm{D}$ en Sistemas de Información geográfica Especialista en Sistema de Información Geográfica. Ingeniera Civil. Universidad Pontificia de Salamanca, campus de Madrid - UPSAM. Paseo Juan XXIII, 3, 28040. Madrid España.

Correo electrónico: sandra.velazcof@gmail.com

${ }^{2} \mathrm{Ph}$.D. en Ingeniería de la Computación, Licenciado en Física. Universidad Pontificia de Salamanca, campus de Madrid - UPSAM. Paseo Juan XXIII, 3,

28040. Madrid - España. Correo electrónico: luis.joyanes@upsam.net

\section{Resumen}

El uso y manejo de la Información Geoespacial (IG) es muy amplio y aplicable en casos como: manejo y administración de los recursos naturales y del medio ambiente; prevención de desastres; ordenamiento del territorio; planeamiento de infraestructuras (transporte, vías, servicios básicos de saneamiento, edificaciones, etc.); seguridad ciudadana; catastro; etc. Todo este tipo de Información que se presta de vital importancia como ayuda en la toma de decisiones tanto para las administraciones públicas como en otros sectores (empresa privada, el sector no gubernamental, los organismos internacionales, el ámbito académico etc.), con el fin de promover el desarrollo sostenible de una región y la mejora de su bienestar social y económico. Esta visión junto con el desarrollo de las telecomunicaciones y la evolución de la Web, ha supuesto un salto específico para las Tecnologías de Información Geográfica (TIGs). El uso de internet multiplica las posibilidades de compartir, distribuir, intercambiar y acceder a los datos. En muchos países, cada vez más la toma de decisiones se sirve del uso de la información geográfica y de las herramientas facilitadas para su manejo, dado un gran impulso a la administración pública en cuanto a la planificación y gestión de sus territorios. Una gran variedad de herramientas y servicios en la Web nos ofrecen la posibilidad de adaptar y adoptar estas tecnologías como herramientas GIS (Geographic Information Systems) que permiten entre otras cosas geolocalizar o georreferenciar nuestros territorios como instrumentos para una adecuada gestión de los mismos. Así mismo, la gran combinación entre Internet y los dispositivos móviles, junto con la tecnología de geolocalización, permite que los usuarios puedan obtener, compartir y consultar información con efecto geográfico a tiempo real. En la actualidad, un gran número de instituciones públicas y privadas se esfuerzan en ofrecer IG a través del servicio de geoportales cuya motivación debe ser lo que demanda la comunidad, procurando dotar a los portales de información geográfica de un nuevo concepto basado en las "necesidades reales de sus usuarios finales".

Palabras clave: Información geoespacial, SIG, geolocalización, georreferenciación, ordenamiento territorial, servicios web, geoportales, IDEs, dispositivos móviles, GPS. 


\section{Abstract}

The use and management of Geospatial Information (GI) is very broad and applicable in cases such as: management and administration of natural resources and the environment; disaster prevention; land use; planning of infrastructure (transport, roads, basic sanitation, buildings, etc.); citizen security; cadastre; etc. All this type of information that is delivered is vital importance to aid in decision making both for public administrations as well as in other sectors (private company, non-governmental sector and international agencies, academia etc.), in order to promote the sustainable development of a region and the improvement of their social and economic welfare. This vision along with the development of telecommunications and the evolution of the Web, has been a specific jump Geographic Information Technologies (TIGs). The use of the Internet multiplies the possibilities to share, distribute, exchange and access to the data. In many countries, increasingly the decision-making is served of the use of geographical information and the tools provided to manage, given a great impetus to the public administration in regard to the planning and management of their territories. A wide variety of tools and services on the Web we offer the possibility to adapt and adopt these technologies as tools GIS (Geographic Information Systems) that allow inter alia geolocalizar or georeference our territories as instruments for the proper management of the same. At present, a large number of public and private institutions are striving to offer IG through the service of geoportals whose motivation should be what the community demand, its efforts to rejuvenate the geographic information portals of a new concept based on the "real needs of its end-users".

Keywords: Geospatial information, GIS, geolocation, georeferencing, land management, web services, geoportals, SDI, mobile devices, GPS.

\section{Introducción}

L as nuevas Tecnologías de la Información (TI) provocan hoy día grandes desafíos organizacionales y una carrera constante hacia la innovación, tanto al interior de las empresas como de las administraciones públicas ya sean estas a nivel nacional, regional o local.

Por otro lado, se dispone de una gran cantidad de Información Geográfica (IG) que se genera desde cualquier ámbito y en particular desde estas administraciones. Es por ello, que para acceder a ésta información, se utilizan una serie de portales Web, que posibilitan un manejo conjunto, interoperable e integrado de la información procedente de distintas fuentes, haciendo necesario el desarrollo de las tecnologías de la información y la aplicabilidad de servicios interoperables, posibilitando así las Tecnologías de la 
No. 1

Información Geográfica (TIGs) como es el caso de los Sistemas de Información Geográfica (SIG, GIS - acrónimo en inglés) y de las Infraestructuras de Datos Espaciales (IDEs).

El nacimiento de las Infraestructura de Datos Espaciales (IDEs) aparece como consecuencia de las dificultades de lograr una interoperabilidad de los datos; compartir información se vislumbra necesario para los organismos con facultad de decisión pero existen dificultades para lograrlo, tal y como lo expresa la Directiva INSPIRE ${ }^{1}$ (Infraestructure for Spatial Information in Europe), de la Comunidad Europea. Lo importante es que las IDEs están basadas en servicios que ofrecen funcionalidades sobre las distintas capas de información. Estos servicios se encuentran estandarizados para garantizar las interoperabilidad del sistema.

Ante la posibilidad de acceder, comparar e integrar los datos a través de Internet entre distintas instituciones y bases de datos, se debe dar solución a ciertas características asociadas a la información geográfica para que ésta logre ser compartida con facilidad. Ante esto, en toda comunicación debe existir un código común que actúe como idioma de intercambio. La Interoperabilidad es "la condición mediante la cual, sistemas heterogéneos pueden funcionar como si formaran parte de un sistema único, y así intercambiar datos y distribuir procesos" [1]. Es decir, la capacidad de los sistemas para intercambiar y utilizar los datos sin gran esfuerzo. Los estándares que hacen esto posible se han elaborado y promulgado por varias organizaciones, incluyendo las Agencias Cartográficas Nacionales, organismos internacionales de normalización, y el Consorcio Geoespacial Abierto (OGC ${ }^{2}$, Open Geospatial Consortium) [2]. La interoperabilidad permite a los sistemas intercambiar datos y ejecutar tareas en conjunto [3], llegando a un punto, en que la información debe ser entendida y útil después de dicho intercambio [4]. El desarrollo de estos estándares y la revolución del software libre o de código abierto han permitido el progresivo acercamiento de la IG al usuario común [5].

La interoperabilidad se consigue cuando las personas, organizaciones o sistemas pueden trabajar juntas. Esto es importante para la información geográfica debido a la exigencia de "compartir información" y procesos en un campo amplio que incluya a diversos tipos de información sobre el mundo con sus características diversas y complejas y los procesos de contratación de un amplio espectro de aplicaciones, personas y organizaciones [6]. La interoperabilidad, cuando se trata de compartir información geoespacial, es más difícil porque la geografía del mundo es tan diversa y compleja, y el hecho de que todos vemos las cosas de manera diferente a causa de nuestra disciplina profesional, nuestra cultura, y/o nuestra visión del mundo a la luz del tema que nos ocupa en un momento determinado.

En la actualidad, tanto el intercambio como la visualización de información por medio de Servicios Web Geográficos basados en la arquitectura de Servicios Web del OGC, permiten la integración de una amplia variedad de servicios "online" de visualización, geoprocesamiento y localización, que facilitan a los SIG, comunicarse unos con otros mediante una variedad de lenguajes $\left(\mathrm{XML}^{3}\right.$, $\mathrm{HTTP}^{4}$ ), lo que indica que dichos sistemas sean capaces de conocer como de utilizar tales servicios. Esta capacidad es posible debido a las reglas establecidas para que dichos servicios hagan pública sus funcionalidades y la forma en que se debe interactuar (como se deben enviar las peticiones de servicio) vía métodos y protocolos abiertos y estandarizados.

'DIRECTIVA 2007/2/CE DEL PARLAMENTO EUROPEO DEL CONSEJO, del 14 de Marzo de 2007, por la que se establece una Infraestructura de Información Espacial en la Comunidad Europea. Disponible en [http://inspire.jrc.ec.europa.eu/] [Consultado en: Junio 6, 2012] ${ }^{2}$ OGC (http://www.opengeospatial.org/)

${ }^{3}$ XML (eXtensible Markup Language (lenguaje de marcas extensible)) ${ }^{4}$ HTTP (Hypertext Transfer Protocol) 
Una forma clara y de fácil consulta y visualización de información geográfica son los visores de información geográfica disponibles en la Web a través de los llamados Geoportales. Dichos visores son herramientas que cumplen las normas y estándares de implementación e interoperabilidad, desarrollados para aquellos usuarios cuyas necesidades no son el análisis, ni la edición sino simplemente: ubicación, localización, consulta y contextualización de información.

Ante esto, y teniendo en cuenta el surgir tecnológico y su constante evolución, quienes tenemos la oportunidad y la capacidad de conocimiento sobre ciertos temas que sirven como sustento a conformar nuevas herramientas que faciliten de cierta manera el desarrollo y crecimiento de un ámbito específico, que en este caso es el ámbito geoespacial y más concretamente con todo aquello vinculado al manejo, integración, análisis, producción y visualización de la información geográfica; encaminamos nuestros esfuerzos en crear, desarrollar, innovar, aplicar y proporcionar, tanto a personas como a entidades (públicas o privadas), herramientas tecnológicas innovadoras que les provea de conocimiento e información de su entorno junto con su ubicación y que a su vez permitan generar beneficios personales, institucionales o colectivos, tal y como se puede advertir y evidenciar con la creación, implantación e inminente implementación del Geoportal IDE Chinácota.

\section{Materiales y métodos}

\section{Conceptualización General}

Es indudable la importancia de los datos geográficos como eficientes instrumentos de soporte a la gestión, principalmente en el ámbito territorial. De esta forma las Tecnologías de la Información Geográfica (TIG) representan un apoyo importante a la hora de gestionar el territorio, de diagnosticar problemas actuales y futuros, o de encontrar las rutas más viables para la toma de decisiones [7].
El objetivo fundamental de estas tecnologías es el de "gestionar y analizar información geográfica o geoespacial" [8]. Los avances en estas tecnologías han conducido a un incremento fenomenal en la cantidad de datos espaciales, a nuevos métodos de análisis para convertir estos datos en información. Se puede decir que el núcleo de estas tecnologías se encuentra en la aplicación de: los Sistemas de Información Geográfica (SIG), los Sistemas de Geoposicionamiento Global (GPS), la Percepción Remota (PR) y las Infraestructuras de Datos Espaciales (IDEs).

La llegada de los Sistemas de Información Geográfica supuso disponer de una herramienta con la que poder realizar análisis con base en la información almacenada en las bases de datos del computador y mostrar un resultado de manera gráfica. Aunque al principio operaban exclusivamente de manera local y no interconectada, los SIG se convirtieron rápidamente en sistemas capaces de proporcionar ayuda en los procesos de toma de decisión, gestión de territorios, gestión de emergencias, gestión de negocios, etc. [9]. Los GIS han pasado de ser una herramienta de cartografía automatizada y gestión de datos a ser una tecnología de análisis con capacidad de manipulación de datos espaciales [10].

Así entonces, los SIG son una herramienta de análisis de información que permiten su almacenamiento espacial de forma coherente y eficiente, facilitando su actualización, manipulación y acceso directo al usuario, y que en efecto, no son solo un programa de ordenador para hacer y almacenar mapas como algunos creen [11].

Los SIG pueden tener sus bases de datos distribuidas en distintos servidores y acceder a ellas mediante redes de comunicación. Esto permite el acceso a la información remota existente, pero para poder mezclar la información que proviene de cada servidor, ésta debe tener ciertas características que permitan la interoperabilidad. Por ejemplo: que los archivos sean compatibles o que cumplan estándares comunes [12].
Enero-Junio 2013 ISSN 0122-820X 
No. 1

\section{Herramientas de SIG como soporte a la planificación territorial}

La Planificación Territorial es un proceso estratégico orientado al futuro, sustentado en criterios racionales para la toma de decisiones. En la actualidad, los Sistemas de Información Geográfica (SIG) forman un campo interdisciplinar, que reúne una gran cantidad de áreas como la Geografía, las Ciencias de la Computación, la Cartografía, la Ingeniería, la Planificación Territorial, etc.

En Colombia, el Ordenamiento Territorial (OT) constituye un instrumento que expresa la dimensión espacial de las políticas sociales, económicas, culturales y ecológicas, con múltiples objetivos, entre los cuales sobresalen el desarrollo socio-económico y equilibrado del territorio con equidad entre hombres y mujeres, mejoramiento de la calidad de vida y de la seguridad ciudadana y humana de sus habitantes, la gestión responsable de los recursos naturales, la protección del medio ambiente y la utilización racional y diferenciada del suelo, entre otros [13]. El OT, se constituye como la carta de navegación para ordenar el territorio, tanto en el suelo urbano como en el suelo rural, pudiéndose expresar en un modelo espacial de ocupación del territorio a consolidar en el largo plazo, acompañado de un conjunto de herramientas que posibilitan su puesta en marcha, a través de la definición de las estrategias territoriales de uso, ocupación y manejo del suelo, en función de los objetivos económicos, sociales, urbanísticos y ambientales de la región.

Los SIG considerados como una herramienta aplicada a integrar y analizar información espacial, pueden ser vistos hasta cierto nivel, como una herramienta de gran poderío para sustentar este tipo de procedimientos. Ciertas características particulares de los SIG que los hacen muy aptos para ser aplicados en un proceso de planificación territorial, son las siguientes: la información procesada en un SIG siempre está relacionada con el territorio. Bases de datos espaciales bien estructuradas favorecen la planificación espacial. Permiten el manejo de altos volúmenes de información. Por su capacidad de ejecutar análisis y modelamientos cartográficos requeridos para proporcionar información relevante en la planificación territorial. Su facilidad de superponer y/o enfrentar diferentes capas de información en diferentes niveles de detalle de manera jerárquica, coincide con la práctica de la planificación espacial. Se puede aplicar para buscar relaciones y cohesión entre diferentes capas de información espacial. Contiene modelos y análisis poderosos que favorecen a la planificación espacial. Su agilidad de editar y adaptar datos propicia flexibilidad en el proceso de planificación. Permite elaborar valoraciones ágiles de los resultados así como hacer presentaciones atractivas para tomadores de decisiones. Dispone de un carácter integrador que se puede aplicar a diferentes datos sectoriales.

Pero para realizar una adecuada gestión del territorio, es necesario definir la localización y características de las cosas del mundo real, del mundo que nos rodea, de manera que se pueda ver, interpretar y analizar la complejidad del medio tanto natural como humano. De esta forma se podría generar información que permita tomar decisiones con el fin de realizar un uso adecuado de los recursos, y de la misma forma optimizar la planificación y la gestión de distintas actividades que contribuyan al desarrollo consensual de la población.

Los Sistemas de Información Geográfica (SIG), son las herramientas que se han desarrollado mundialmente para "ayudar" a llevar a cabo estas tareas. Las ventajas que estos ofrecen para la gestión del territorio se basan en su capacidad de integrar gran cantidad de información de diversas fuentes, permitiendo su consulta y análisis de forma rápida y directa, optimizando el tiempo, el coste y los resultados [14]. Gracias a estas capacidades los SIG se han convertido en herramientas indispensables para muchos sectores de actividad tanto públicas como privadas.

Por otro lado, los mapas se ha convertido en algo habitual, y cualquier recurso puede ser 
localizado sobre uno de ellos. Geolocalizar es como bien dice su palabra, localizar un punto es un lugar concreto, en un mapa.

Actualmente es muy común hablar de geolocalización, que consiste simplemente en conocer la ubicación geográfica de algo o de alguien de manera automática a partir de unas determinadas coordenadas. El termino geolocalización, también denominado georreferenciación implica el posicionamiento que define la localización de un objeto en un sistema de coordenadas determinado. Es un concepto relativamente nuevo, que ha proliferado de unos dos años a esta parte y que hace referencia al conocimiento de la propia "ubicación geográfica" de manera automática y en lo posible en tiempo real. Este término, comenzó a crecer a un ritmo acelerado con la evolución de la tecnología móvil y más específicamente con el uso de los smartphone (teléfonos móviles de última generación), que de un tiempo para acá traen integrados receptores GPS que mediante la red de satélites que rodea al planeta, nos entregan la ubicación de cualquier punto del globo.

Aun así, aunque existen un gran número de instrumentos que nos faciliten determinar ubicación en forma de coordenadas (latitud, longitud), Internet, nos ofrece una herramienta conocida globalmente como Google Maps ${ }^{5}$ que nos ofrecen geolocalización sin necesidad de disponer de un dispositivo GPS, navegador o móvil, simplemente con conexión a Internet, desde el navegador (Internet Explorer, Chrome, Firefox, Opera), usando el $\mathrm{API}^{6}$ de geolocalización que tiene Google. De tal manera que si necesitamos ir de un sitio (lugar) a otro, gracias a esta tecnología, solo tenemos que decir a dónde vamos, pues por medio del servicio de geolocalización ya se sabe dónde estás y posiblemente a donde llegar. Sin duda Google Maps es una de esas herramientas que han evolucionado el mundo de la geolocali- zación. Google Maps, como servicio gratuito forma parte de Google Earth 7 , y entre otras cosas nos permite visualizar imágenes en 3D del planeta y combina imágenes del satélite y mapas, permitiendo que sus usuarios vean a escala un lugar específico del globo.

Aun así, con la facilidad de poder contar con esta herramienta, es significativamente necesario optar por buscar posicionamiento con dispositivos como el GPS, en parte porque a ciertas zonas del planeta se hace más complicado obtener una imagen de satélite, por diversas razones (meteorológicas, pésima resolución, no paso del sensor, etc.), o porque el API de geolocalización de una zona en común no este del todo o nada funcional, ocasionando de tal manera, que servicios como los que presta Google sean del todo desaprovechados.

En consecuencia, los SIG, representan un papel importante como herramienta para fomentar la ejecución de ciertas acciones (captura, recopilación e integración de datos; sectorización de zonas, limitación de zonas, distribución de zonas, elaboración de planes de manejo, localización, etc....) que impliquen intervención en el territorio. Aun así, el éxito de la aplicación de estas tecnologías, básicamente dependerá de una apropiada capacitación del recurso humano y de la selección de apropiados ambientes operacionales que conlleven a valorar la capacidad y potencialidad de estas, tanto en el ámbito de captura y organización de los datos, como en análisis y presentación (visualización) de la información como soporte a la toma de decisiones.

Sin embargo, aunque un SIG pueda obtener la IG de sus servidores dispersos, para poder compartir toda la IG disponible sobre un territorio hace falta que existan acuerdos entre los propietarios de esa información para que pueda ser compartida y programas capa-

${ }^{5}$ Google Maps, página principal [maps.google.com/]

${ }^{6}$ Interfaz de programación de aplicaciones API (del inglés Application Programming Interface)

${ }^{7}$ Google Earth, página principal [earth.google.com/] 
No. 1

ces de mostrar la información compartida.

Es aquí es donde intervienen las Infraestructuras de Datos Espaciales (IDEs).

Figura 1: Qué es una IDE. [Fuente: GeoBolivia]

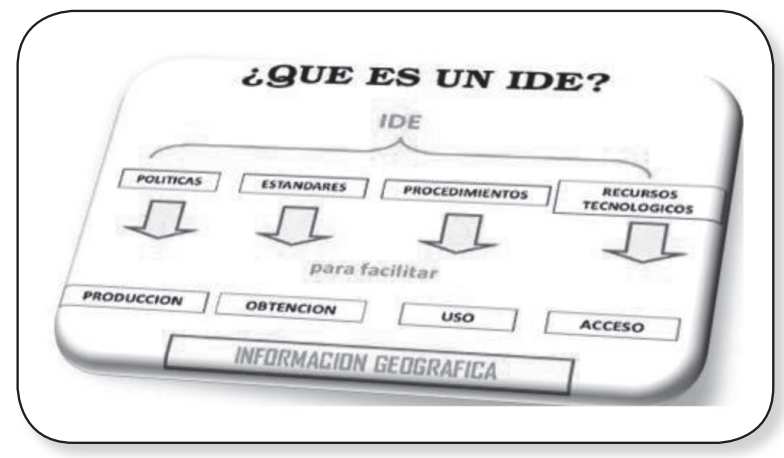

Como se había mencionado con anterioridad, el nacimiento de la Infraestructura de Datos Espaciales surge como consecuencia de las dificultades presentes por lograr una Interoperabilidad de los datos. Así entonces se define una Infraestructura de Datos Espaciales (IDE) como "un sistema informático integrado por un conjunto de recursos (catálogos, servidores, programas, datos, aplicaciones, páginas Web,...), que permiten el acceso y la gestión de conjuntos de datos y servicios geográficos descritos a través de sus metadatos (mapas, ortofotos, imágenes de satélite, topónimos,...), disponibles en Internet, que cumple una serie de normas, estándares y especificaciones que regulan y garantizan la interoperabilidad de la información geográfica (normas, especificaciones, protocolos, interfaces,...). Los recursos permiten que un usuario, utilizando un simple navegador, pueda utilizarlos y combinarlos según sus necesidades" [15] (Véase Figura 1).

La creación y establecimiento de una IDE,

se justifica en base fundamentalmente a dos necesidades que deben ser resueltas [16]: 1) la de obtener fácil acceso a la información geográfica. Se debe acceder fácil, cómoda y eficazmente a los datos geográficos existentes. La IG, que es un recurso de costosa producción y de difícil acceso (formatos, modelos, políticas de distribución, falta de información,...) debe dejar de serlo; y 2) la reutilización de la información geográfica. La IG debe poder reutilizarse una vez que ha servido para el proyecto para el cual ha sido adquirida. Se debe reutilizar la IG generada en un proyecto para otras finalidades diferentes, dado el alto coste de su producción.

Buscando la resolución de estas dos necesidades, el termino IDE es utilizado para mencionar la colección de tecnologías, políticas y estructuras institucionales que facilitan la disponibilidad y acceso a la IG, ofreciendo la posibilidad de acceder a datos espaciales "heterogéneos" y distribuidos a través de este conjunto de políticas, reglas comunes y estándares, facilitando la interconexión de la información espacial de una manera interoperable [17].

La información geográfica que gestiona una IDE puede estar en forma de ortofotos, imágenes de satélite, mapas, nombres geográficos, capas de información de un SIG, etc. (Véase Figura 2). Así mismo, la IG a la que se quiere acceder debe ser acorde con ciertas normas y estándares y los recursos informáticos con especificaciones, protocolos e interfaces que garanticen la interoperabilidad. El ideal de los estándares $^{8}$ de la información geográfica, es el mundo en el que todos se beneficien de dicha

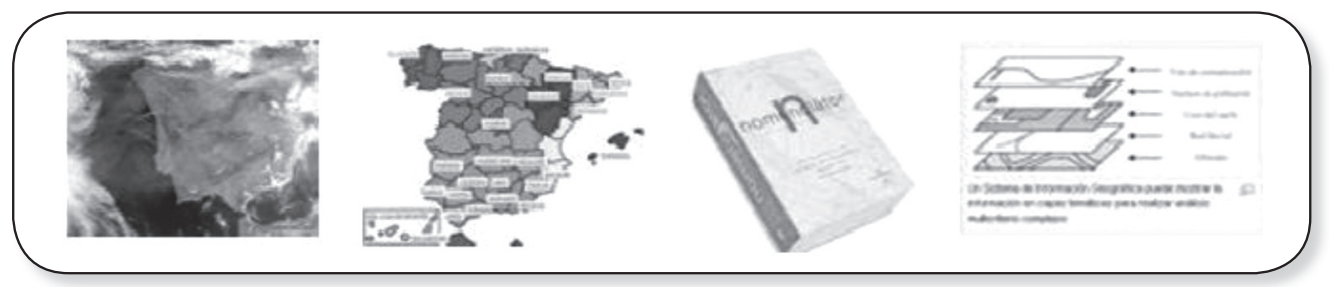

Figura 2: Información geográfica gestionada en una IDE. [Fuente: IGN]

\footnotetext{
${ }^{8}$ La ISO (International Organization for Standardization), define un estándar como: "un documento que establece los requisitos, especificaciones, directrices o características que pueden ser usados constantemente para asegurar que los materiales, productos, procesos y servicios sean aptos para su propósito". Página principal [http://www.iso.org/iso/home.html] [Consultado en: Junio 6, 2012]
} 
información y que los servicios estén siempre disponibles a través de cualquier red, aplicación o sistema.

Gracias a la fuerte expansión de las IDEs se ha conseguido que una gran parte de la información geográfica existente pueda ser consultada, estudiada, analizada y/o tratada por cualquier usuario a través de un simple navegador Web. Lo que, sin duda, está favoreciendo a la accesibilidad y universalización de un sinfín de datos geográficos que hasta hace poco sólo estaban al alcance de unos pocos afortunados [18]. Un geoportal es el punto de acceso a los contenidos de las Infraestructuras de Datos Espaciales (IDEs) [19]. Actualmente, podemos apreciar de forma clara y sencilla, que tanto la consulta como visualización de IG es facilitada por visores de información geográfica disponibles en la Web a través de los geoportales. Estos visores que se muestran como herramientas que cumplen las normas y estándares de implementación e interoperabilidad, son desarrollados para aquellos usuarios cuyas necesidades no son el análisis ni la edición, sino simplemente la ubicación, localización, consulta y contextualización de información.

\section{Portales Geográficos o Geoportales}

Aunque existen múltiples definiciones sobre lo que son los servicios Web, una posible sería hablar de ellos como un conjunto de aplicaciones o de tecnologías con capacidad para interoperar en la Web. Estas aplicaciones o tecnologías intercambian datos entre sí con el objetivo de ofrecer unos Servicios [20]

El desarrollo y mantenimiento de un geoportal, permite tanto a productores como a usuarios de la información geográfica, el acceso, la disponibilidad, el uso, la reutilización y el intercambio de datos espaciales, así como el desarrollo de aplicaciones específicas online para la satisfacción de necesidades puntuales de la sociedad, convirtiendo a la información geográfica en un bien de uso y beneficio común [21].
Así pues, un geoportal es un sitio Web cuyo objetivo es ofrecer al usuario, de forma práctica e integrada, el acceso a una serie de recursos y servicios basados en IG, resolviendo la conexión física y funcional entre los almacenes de datos geográficos y los usuarios de información geográfica [22].

Los datos que puede ofrecer un geoportal pueden ser de lo más variado, y definen el tipo de geoportal que queremos desarrollar: turístico, de información urbanística, comercial, etc. Un geoportal utiliza la red para facilitar el descubrimiento, acceso y visualización de datos geoespaciales, por medio un navegador estándar de internet, favoreciendo la interoperabilidad, integración, e intercambio de información entre ciudadanos, Instituciones o agentes sociales. Con el auge de las IDEs, estos servicios han aumentado considerablemente su potencialidad, tanto por la prestación del servicio (desarrollos sobre servicios Web Geográficos estandarizados OGC: WMS (Web Map Service), WFS (Web Feature Service), WCS (Web Coverage Service), Catálogos,...) como por la posibilidad de ser invocados tanto desde el portal propio como desde otros externo.

\section{Características de un Geoportal}

Un buen geoportal debe cuidar aquellos aspectos que hacen más eficaz su utilización por todo tipo de usuario, en particular [23]:

Universalidad, un geoportal debe ser multilingüe y tener, al menos, una versión en inglés; debe ser además visible desde los sistemas operativos más usuales (Windows, Unix, Linux, Macintosh,...) y utilizando los navegadores más frecuentes (Internet Explorer, Firefox, Opera, Mozilla, Safari, Netscape,...)

Usabilidad, un geoportal debe ser cómo, fácil de utilizar, auto-explicativo y sencillo.

Accesibilidad, para facilitar el acceso a usuarios discapacitados, al menos a las páginas estáticas, dadas las dificultades 
No. 1

Enero-Junio 2013 ISSN 0122-820X técnicas de hacer accesibles los contenidos cartográficos.

Diseño, de manera que sea atractiva, ordenado, seductor y trasmita los valores que el proyecto considere más importante.

Disponibilidad, del geoportal y de las aplicaciones cliente en él integradas.

Rendimiento, de las aplicaciones cliente, de manera que la respuesta sea rápida incluso en momentos de alta demanda y con gran número de usuarios concurrentes. El internauta típico abandona cualquier portal Web que tarde en responder más de unos pocos segundos.

\section{Uso de los Geoportales}

Tabla 1: Uso de los Geoportales. [Fuente: Elaboración propia]

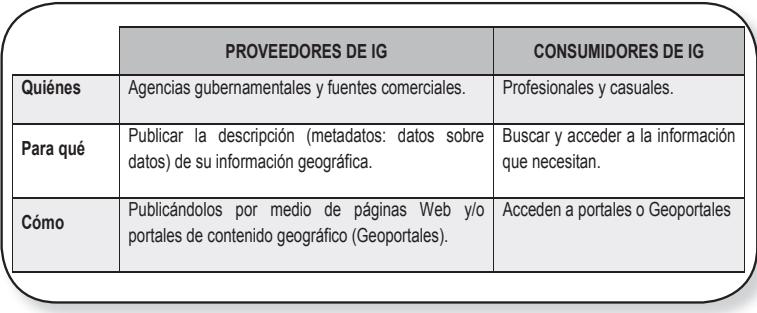

\section{Ventajas que ofrece un Geoportal}

- Cooperación interinstitucional.

- Planeación estratégica.

- Minimizar la duplicación de esfuerzos.

- Socialización de los datos/información.

- Resaltar el valor de la información geográfica.

- Información actualizada y continua.

- Análisis para tomadores de decisiones.

- Realización y actualización de metadatos.

- Única base nacional, regional, local.

- Definición de políticas.

- Definición de estándares.

\section{Software de aplicación}

Son múltiples las herramientas software que se utilizan para el desarrollo e implementación de aplicaciones en el contexto de una IDE, y que deben cumplir con los estándares del Open Geospatial Consortium (OGC) para que puedan integrarse y ser accesibles a través de un geoportal Web (Véase Figura 3).

Hay que destacar que entre las múltiples herramientas se encuentran tanto software propietario como software libre.

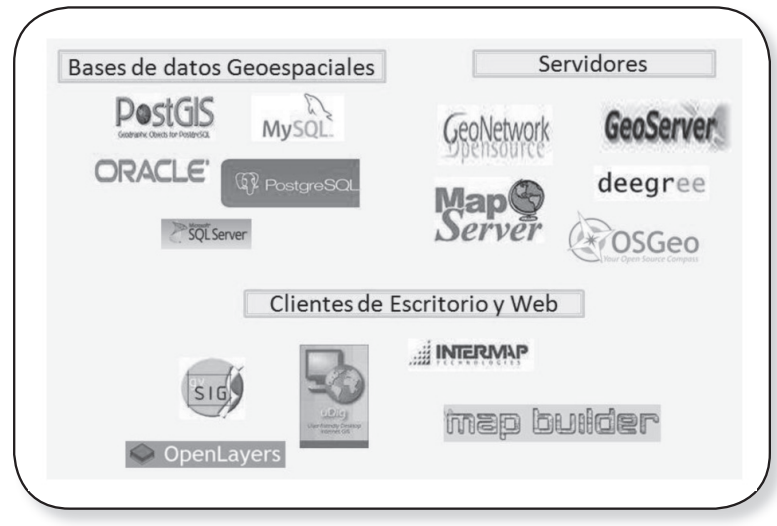

Figura 3. Ejemplo Software IDE. [Fuente: Elaboración propia]

\section{Resultados}

\section{Propuesta para la creación del Geoportal IDE del municipio de Chinácota}

Chinácota, es un Municipio ubicado en el departamento de Norte de Santander, distante 39,7 km de su capital Cúcuta y 586 $\mathrm{km}$ de Bogotá (capital de Colombia). El territorio municipal se encuentra ubicado en el valle de la cordillera oriental, a 7@-37' latitud norte y 72³6'; longitud oeste, del Meridiano de Greenwich. El municipio de Chinácota pertenece a la subregión sur-oriental del departamento Norte de Santander, con una extensión total de $166.64 \mathrm{Km}^{2}$, extensión área urbana: $3.8 \mathrm{Km}^{2}$, extensión área rural: 162.84 $\mathrm{Km}^{29}$.

\footnotetext{
${ }^{9}$ Datos del sitio oficial, disponible en [http://www.cucuta-nortedesantander.gov.co/nuestromunicipio.shtml?apc $\left.=\mathrm{mIxx}-1-\& \mathrm{~m}=\mathrm{f}\right]$
} 
Teniendo en cuenta que la información geográfica juega un papel muy importante en el desarrollo de la sociedad, esta debe ser vista como un recurso fundamental para desarrollo sostenible de una organización, institución pública o privada. Para el caso de las administraciones públicas, los entes municipales deben considerar de vital importancia los avances tecnológicos que conduzcan a facilitar al ciudadano el acceso a la información pública, como una oportunidad para potenciar las Infraestructuras de Datos Espaciales con información correspondiente al ámbito local, permitiendo así, que municipios como Chinácota, puedan estar en una posición de privilegio para su potencial incorporación en la IDE de Colombia y poder convertirse en una administración de referencia en este ámbito y constituirse como experiencia piloto para el entorno administrativo local y regional.

La puesta en práctica de un proyecto de Infraestructura de Datos Espaciales IDE, se materializa a través de un geoportal que ofrezca como mínimo los siguientes tres servicio: servicio de visualización (que permita la visualización de los datos a través de servicios Web y, opcionalmente, su consulta), servicio de localización (que posibilite la búsqueda de conjuntos de datos y servicios a través del contenido de sus metadatos) y servicio de Catalogo (que permita la localización en un mapa a través de un nombre geográfico).

El desarrollo y mantenimiento de un geoportal, permite tanto a productores como a usuarios de la información geográfica, el acceso, la disponibilidad, el uso, la reutilización y el intercambio de datos espaciales, así como el desarrollo de aplicaciones específicas online para la satisfacción de necesidades puntuales de la sociedad, convirtiendo a la información geográfica en un bien de uso y beneficio común [24].

No podemos negar que una Infraestructura de Datos Espaciales es mucho más que un geoportal, pero en este proyecto nos enfocaremos especialmente a ésta herramienta. El propósito es poder identificar un modelo de desarro- llo que cumpla con todos los requerimientos tanto a nivel internacional como nacional en cuanto a interoperabilidad, producción, manejo y uso de la información geográfica.

\section{Ámbito de aplicación del OT en el munici- pio de Chinácota}

Como muchos municipios en Colombia, Chinácota no está exento de necesidades de índole económico, político, social, de salud, de infraestructura, la escasez de información, dificultad de localización, del desconocimiento de muchas de sus características, etc., que obligan a buscar alternativas de solución y de toma de decisiones que den cómo resultado labores de prevención, mantenimiento y/o correctivos útiles como herramientas idóneas para las demandas presentadas por el municipio y la región.

El Acuerdo № 009 del 10 de Mayo de 2003 "por el cual se adopta el Esquema de Ordenamiento Territorial del Municipio de Chinácota para el período 2003 - 2014 y se dictan otras disposiciones", es el que enmarca el Plan de Ordenamiento Territorial (POT) del municipio.

En consecuencia, y teniendo en cuenta las consideraciones presentes en el Acuerdo No. 006 del 11 de Julio de 2007, "[... por el cual se adopta el proyecto de revisión, modificación y ajuste del EOT (Esquema de Ordenamiento Territorial) del Municipio de Chinácota según modificación excepcional de Normas Urbanísticas", el cual alude entre consideraciones, que "[...] el municipio de Chinácota debe adoptar la revisión parcial, ajuste y nuevas disposiciones de su Esquema de Ordenamiento Territorial en cumplimiento de lo dispuesto Ley 388 de 1997 en su artículo 9 como un instrumento básico para desarrollar el proceso del Ordenamiento del Territorio municipal, definiéndolo como un conjunto de objetivos, directrices, politicas, estrategias, metas, programas, actuaciones y normas adoptadas para orientar y administrar el desarrollo físico del territorio y la utilización del suelo. Por lo que, se hace necesario realizar una revisión general a las normas urbanísticas del EOT, debido a que este presenta conflictos o dualidad en su interpretación por no presentar unas
Enero-Junio 2013 ISSN 0122-820X 
No. 1

Enero-Junio 2013 ISSN 0122-820X normas claras, las cuales han permitido que este instrumento de planificación se convierta en un tropiezo para el desarrollo armónico de la ciudad" y que "[...] además, se deben corregir impresiones normativas y cartográficas relacionadas con las áreas y usos del suelo municipal, las cuales dificultan la acción de planificación en el territorio, siendo indispensable realizar cambios en los usos del suelo, debido a que no están cumpliendo con su función social y por el contrario dificultan el proceso de crecimiento y desarrollo del municipio".

Ante esta situación, se "propone" la implantación de un sistema capaz de aportar ideas, de visualizar formas, de promover estrategias, de agilizar tácticas, consolidando el carácter de infraestructura básica de desarrollo que posibilite una mejora en el proceso de toma de decisiones de cualquier actuación sobre el territorio, sustentando así la conformación y creación de un geoportal, como herramienta capaz de establecer acciones apropiadas para el crecimiento y desarrollo del municipio. La actual administración, en cabeza de su alcalde y de la oficina de Planeación y Desarrollo Municipal, buscan estrategias que combinen el desarrollo y la utilización de este tipo de herramientas y que admitan que tanto administradores como personas del común, se orienten junto con las políticas, estrategias, objetivos, acciones y actuaciones planteadas por los diferentes entes municipales en el EOT (Esquema de Ordenamiento Territorial), en sus componentes general, urbano y rural, como instrumento que permita estimular el proceso de crecimiento y desarrollo del municipio de Chinácota como Paraíso AgroTurístico.

\section{Riesgos y obstáculos}

La Información Geográfica producida en las instancias de los diferentes sectores de la comunidad de Chinácota, no es de fácil disposición ni acceso, ni mucho menos completa ni organizada para ser compartida con el mínimo de calidad dictaminado por las agencias tanto nacionales como internacionales para tales efectos.
Esto conlleva a encontrar una serie de inconvenientes:

- Información no catalogada.

- Dificultad para encontrar los datos reque ridos: ¿Quién los tiene? ¿Como los requiero?

- Desconocimiento del origen, calidad y linaje de los datos conseguidos.

- Circulación de copias y versiones diversas de datos.

- Falta de transferencia tanto de datos como de conocimiento.

- Escasez de recurso humano capacitado.

- Escasez de recursos tecnológicos (software, equipamiento adecuado, y otros).

- Falta de protocolos y estándares, y a su vez esto conlleva a que se presente:

- Incoherencia en la Información, sin articulación o integración.

- Duplicación de esfuerzos.

- Duplicación de costos.

- Falta de datos adecuados y precisos para la toma de decisiones.

- Falta de servicios de información geográfica.

\section{Objetivos del Geoportal IDE Chinácota}

Los objetivos del Geoportal, como componente de la IDE del Municipio de Chinácota, son:

- Conseguir una mayor integración de las iniciativas relacionadas con el Esquema de Ordenamiento Territorial del municipio, con la mínima dificultad y coste posible, utilizando para ello tecnología libre y consolidada internacionalmente.

- Permitir el descubrimiento de los conjuntos de datos y servicios geográficos de la IDE del municipio de Chinácota que se vayan incorporando y sean accesibles desde Internet.

- Promover la difusión y reutilización de la información geográfica y los geoservicios.

- Ofrecer en la medida de lo posible su funcionalidad en base a geoservicios que cumplan las normas, estándares y especificaciones que regulan y garantizan la inte- 
roperabilidad de la información geográfica.

- Proporcionar funcionalidad de interés general (visor de información, catálogo de datos, descarga de datos, etc.).

- Dar a conocer las novedades o noticias referentes a la IDE del municipio de Chinácota y a la disponibilidad en internet de nuevos conjuntos de datos o geoservicios.

Fase de análisis: identificación y selección de alternativas

Con la exploración de una muestra significativa de geoportales IDE (en especial IDEs de España y de Colombia), acusaremos un patrón básico para la conformación y funcionamiento de los geoportales de IDEs que actualmente ofrecen IG, servicios y aplicaciones que han sido desarrolladas para facilitar o ejecutar tareas específicas pertinentes a la gestión y utilización de los datos espaciales. Con ésta labor, se pretende determinar un patrón común a todos los geoportales explorados, que sirva como indicativo de soporte a considerar para la "implantación de un Geoportal IDE en el municipio de Chinácota, Norte de Santander, Colombia". El propósito es generar un modelo, una referencia básica y sencilla que sirva de base y de guía para la "creación e implementación del primer Geoportal de la Infraestructura de Datos Espaciales del Municipio de Chinácota (que se denominará Geoportal IDE Chinácota), por las autoridades competentes, y en conformidad con las iniciativas desarrolladas a nivel nacional e internacional para tal fin, pero adaptables a la propia realidad política, social y económica del municipio y la región.

Se ha realizado un estudio concienzudo de documentación, visitas a sitios Web, revisión de informes estratégicos, revisión de estándares de interoperabilidad y normas de obligado cumplimiento, en definitiva una amplia exploración y análisis de productos análogos, conexos al tema de investigación, fundamentalmente IDEs nacionales (de España y de Colombia), regionales como aquellas IDEs internacionales que pueden servir de referencia.

Enero-Junio 2013 ISSN 0122-820X

Para lograr concretar un modelo de trabajo razonable, se determinaron las necesidades principales en cuanto al funcionamiento y características tecnológicas del sistema, así como el análisis de la información existente para alimentar la base de datos geográfica. Este análisis permitirá a los gestores públicos contar con criterios claros para la fase de implementación completa del sistema y prepararse en función de los requerimientos tecnológicos.

Análisis del software necesario. En los últimos años, tanto el software libre como de código abierto, cobran cada vez más fuerza como alternativa real y posible ante el software propietario. El software libre y abierto permite a los usuarios acceso al código fuente, modificarlo, corregirlo, mejorarlo, adaptarlo y redistribuirlo en función de sus necesidades. Cuando se tiene un modelo de código abierto, se previene entre otras cosas, el reinventar opciones, duplicar trabajo, reducir tiempo de distribución; permitiendo que el trabajo del Software ya hecho pueda ser reutilizado en otras actividades.

Sistemas gestores de bases de datos. Una base de datos se muestra como un conjunto de datos relacionados entre sí. Los Sistemas de Gestión de Bases de Datos (SGBD) son un tipo de software muy específico, dedicado a servir de interfaz entre la base de datos, el usuario y las aplicaciones que la utilizan, y que permiten almacenar y posteriormente acceder de forma rápida y estructurada a la información contenida en una base de datos. A partir de la utilización de un Sistema Gestor de Bases de Datos (SGBD), se integra la IG, junto a información alfanumérica asociada y otros tipos de información.

Análisis sobre el servidor web. Un servidor web será en encargado contestar todas aquellas peticiones de ejecución que requiera un cliente o un usuario, de forma adecuada, entregando como resultado ya sea una página Web o cualquier otro tipo de información de acuerdo a los comandos solicitados. 
No. 1
Análisis sobre el servidor de mapas. Los servidores de mapas permiten la interacción con la información espacial almacenada en servidores de datos espaciales accesibles vía Web. Un servidor de mapas se encarga de atender las peticiones del servidor web para acceder a la información geográfica y desplegar la misma a los clientes del sistema. El desarrollo de los servidores de mapas ha sido paralelo al desarrollo de aplicaciones en internet y a su integración con tecnologías de SIG, por lo cual existe una amplia variedad de posibilidades y desarrollos.

\section{Transferencia tecnológica final de software libre/abierto}

Las aplicaciones y tecnologías propuestas para la implantación de la arquitectura del geoportal (Véase Figura 4), se sustentan completamente en software libre. Cabe mencionar que el Sistema Operativo para la instalación de los servidores de aplicaciones y bases de datos dependerá directamente del sistema disponible (Windows, GNU/Linux) en la alcandía municipal (Ayuntamiento). En lo correspondiente a las aplicaciones, se proponen las siguientes:

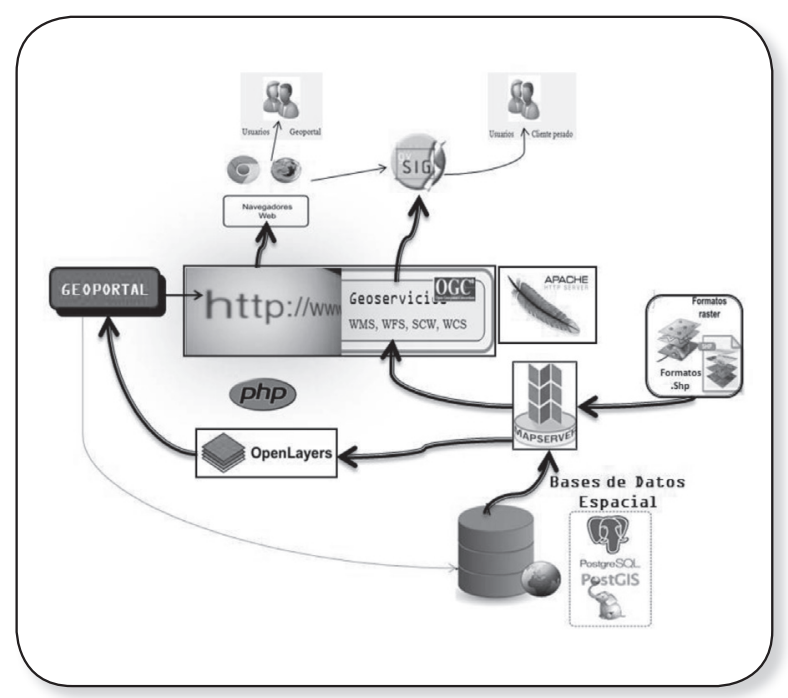

Figura 4. Arquitectura y Tecnologías del Geoportal IDE Chinácota. [Fuente: Elaboración propia]

Servidor de bases de datos. De acuerdo al análisis realizado sobre el sistema gestor de bases de datos (entre MySQL ${ }^{10}$ y PostgrSQL ${ }^{11}$ ), se considera que PostgreSQL resulta ser la mejor opción como SGBD junto con la extensión espacial PostGIS ${ }^{12}$. Este sistema gestor de bases de datos objeto-relacional, con más de 16 años de desarrollo activo, en donde su estabilidad, potencia, robustez, facilidad de administración e implementación de estándares se exponen como las características más destacables durante su desarrollo. PostgreSQL funciona muy bien con grandes cantidades de datos y una alta concurrencia de usuarios accediendo a la vez al sistema, mostrándose hoy en día como el sistema libre más avanzado, fiable y con gran diferencia, soporta la mayoría de las transacciones SQL, control concurrente e integridad de datos. La extensión espacial PostGIS, es un proyecto separado que ofrece el soporte de objetos geográficos y funciones geométricas y topológicas para el tratamiento de los datos espaciales basados en estándares OGC (Open Geospatial Consortium). El Sistema Gestor tiene como funciones principales las siguientes: 1) almacenar estructuradamente y segura todos los datos geográfico vectoriales y alfanuméricos existentes en para el sistema, y 2) permitir una recuperación eficiente de los datos por medio del lenguaje de consulta SQL, así como facilitar las funciones espaciales y de acceso a las geometrías.

Servidor de mapas. Se propone la plataforma de código abierto MapServer ${ }^{13}$, (sobre GeoServer $^{14}$ y MapGuide ${ }^{15}$ ) como aplicación intermedia que permita servir los datos espaciales a través de Servicios Web geográficos (WMS, WFS) a los clientes del sistema. MapServer, originalmente desarrollado por la Universidad de Minnesota, permite el acceso a la base de datos espacial y publicación de la información geográfica de acuerdo a estándares del OGC (Open Geospatial Consortium). Este Servidor de mapas posibilita la integración de todas las fuentes de datos geográficos ráster y vectorial (modelos de datos de un SIG) del sistema, para proporcionar la renderización (proceso de generar una imagen desde un modelo), ya sea como imágenes georreferenciadas o vectores a los visores Web de mapas en la aplicación, o a los clientes que soporten 
estos estándares.

Desde este punto de vista, MapServer brinda la suficiente estabilidad, desempeño, flexibilidad y escalabilidad para el desarrollo del módulo de información geográfico del Geoportal, con las ventajas que representa el código abierto en costo y adquisición junto con la libertad para modificarlo y utilizarlo.

Servidor web. Se considera que la aplicación intermedia encargada de comunicar las peticiones del cliente con las respuestas de los servidores de aplicaciones es Apache HTTP Server $^{16}$, por ser uno de los más potentes del mercado, ofreciendo una perfecta combinación entre estabilidad y sencillez.

Por otro lado, corre en una multitud de Sistemas Operativos, lo que hace de este prácticamente una herramienta universal. Es una tecnología gratuita de código abierto. Es un servidor altamente configurable de diseño modular. Es muy sencillo ampliar sus capacidades. Trabaja con una gran cantidad de lenguajes. Permite personalizar la respuesta ante los posibles errores que se puedan dar en el servidor.

Servicio web de mapas. Se considera la aplicación $g v \mathrm{SIG}^{17}$ es en la actualidad una de las mejores herramientas para Sistemas de Información Geográfica (SIG) desarrollado en software libre. Entre sus múltiples funcionalidades se encuentra la posibilidad de conectar con Servidores que ofrecen WMS, permitiendo la visualización de la imagen cartográfica con la ventaja de poder mostrar la leyenda con múltiples niveles de desagregación. Aparte, combina la visualización con las funcionalidades de una herramienta SIG: posibilidad de combinar capas, de montar nuevas capas teniendo el WMS como referencia, de obtener información sobre los elementos identifi-

\footnotetext{
${ }^{10} \mathrm{MySQL}$, página principal del proyecto [www.mySQL.com]

${ }^{11}$ PostgreSQL, página principal del proyecto [www.postgreSQL.org]

${ }^{12}$ PostGIS, página principal del proyecto [http://postgis.refractions.net/]

${ }^{13}$ MapServer, página principal [www.mapserver.org/]

${ }^{14} \mathrm{Geoserver,} \mathrm{página} \mathrm{principal} \mathrm{del} \mathrm{proyecto} \mathrm{[geoserver.org/]}$

${ }^{15}$ Mapguide, página principal del proyecto [mapguide.osgeo.org/]

${ }^{16}$ Apache HTTP Server, página principal del proyecto [www.apache.org/]

${ }^{17} \mathrm{gvSIG}$, página principal del proyecto [www.gvsig.org/]
}

cados en el propio Servicio WMS (GetFeatureInfo), etc. gvSIG permite al usuario publicar información geoespacial y metadatos a través de Servicios Web estándares OGC, desde la propia interfaz de guSIG y sin necesidad de hacerlo directamente sobre el software del servidor correspondiente.

\section{Viabilidad del Geoportal para el Municipio de Chinácota}

Antes de la puesta en marcha de cualquier proyecto y asumir el riesgo inherente a su desarrollo, es imprescindible realizar un análisis para comprobar su viabilidad económicofinanciera-funcional, de tal forma que los resultados obtenidos nos permitan concluir si es conveniente llevar a cabo dicho proyecto, si hay que realizar alguna modificación o conviene abandonar la idea.

Viabilidad económica. Se dice que un proyecto cual sea, demostrará ser viable económicamente, siempre que se obtenga de él, una rentabilidad (utilidad o beneficio) positiva y aceptable. Por lo tanto, económicamente el proyecto de Geoportal IDE para el Municipio de Chinácota, ha demostrado ser beneficioso, ya que éste, al ser conformado en su totalidad con software libre o de código abierto, no conlleva en cuanto a infraestructura ningún cargo económico. No obstante, cabe mencionar que en algún momento de su etapa de implementación, seguramente conllevará algún coste económico en cuanto a personal, requerimiento de equipos (ordenadores), talleres de actualización, capacitación, mantenimiento, etc., costos considerados no tan altos como los beneficios que se pueden generar.

Viabilidad financiera. Se dice que un proyecto será viable desde el punto de vista fi-
Enero-Junio 2013 ISSN 0122-820X 
No. 1

Enero-Junio 2013 ISSN 0122-820X nanciero, siempre y cuando, en todo momento, demuestre contar con recursos suficientes para poder hacer frente a las obligaciones de pago contraídas en su desarrollo. En nuestro caso como el desarrollo del Geoportal IDE del Municipio de Chinácota, arranca sin ningún coste económico (infraestructura del Geoportal), esto hace que los recursos que se puedan invertir en el mismo sean lo suficientemente cómodos. No obstante, cabe mencionar que para el buen funcionamiento del proyecto seguramente se deberán invertir nuevos recursos al sistema, todo dependerá del trabajo alcanzado por este.

Viabilidad funcional. Se dice que la viabilidad funcional de un proyecto es la capacidad de desarrollo del mismo tanto a nivel técnico, operativo como humano. Ante esto, una vez implementado el Geoportal, se establecerán una serie de talleres, seminarios, y demás actividades pertinentes a capacitar personal idóneo para tal fin. Así mismo, se considera la disponibilidad total de la administración pública para poner en funcionamiento la herramienta.

\section{Conclusiones}

Las nuevas tecnologías facilitan una serie de herramientas dispuesta en la Web de forma libre, que facilitan el manejo de Información geográficamente referenciada como instrumentos para la adecuada planificación y gestión del territorio. Un geoportal puede mostrarse como un "servicio" de consulta pública y si es preciso universal, y en lo posible de carácter gratuito, ofreciendo las posibilidad de geolocalizar servicios, vías, negocios, parcelas, carreteras, caminos, ríos, montes u otro tipo de parajes, realizar croquis y mapas de localización, obtener recorridos, capturar datos Georreferenciados de instalaciones deportivas, agrícolas o referencias catastrales, etc.

Un geoportal puede ser visto como una herramienta de clase mundial, una herramienta que permite no solo a los administradores, a los ciudadanos locales sino foráneos, poder ver y conocer información acerca de una región como recurso turístico, económico, social, laboral, etc. En su faceta más técnica, el geoportal dará acceso controlado a una Infraestructura de Datos Espaciales (IDE), desarrollada en tecnologías Open Source (software libre/abierto), con información geográfica relevante del municipio de Chinácota (Colombia) y que actualmente se encuentra dispersa en las diferentes secciones de la administración municipal (infraestructura, acueducto, electricidad, agropecuaria,...), de forma que pueda ser compartida y contrastada tanto por profesionales instituciones, como por usuarios del común (comunidad).

El hecho es que los geoportales están ahí, esperando a ser consultados y utilizados por sus usuarios, sean estos especialistas o no el tema. Pero para que el usuario final sea el mayor beneficiario de estas herramientas, se debe precisar que el acceso a los mismos sea lo más transparente y claro posible, que no confunda, que sea fácil de interpretar, en definitiva que el usuario pueda disfrutar de un modo muy pactico del uso y posibilidades de estas herramientas.

En la actualidad, un gran número de instituciones públicas y privadas se esfuerzan en ofrecer información geográfica a través de un servicio de geoportal, sin embargo, es preciso considerar que en muchos casos, la prestación de estos servicios no se ciñe a una necesidad tan real de una comunidad, sino posiblemente de una disposición política producto justamente de una cierta competencia institucional. Ciertamente, como motivación debería ser lo que demanda la comunidad, pues se debería procurar dotar a los portales de información geográfica de un nuevo concepto basado en las "necesidades reales de los usuarios finales".

Esta premisa se ha podido constatar como verdadera al explorar el Esquema de Ordenamiento Territorial (EOT), del municipio de Chinácota, así mismo, como en la escasa por no decir "nula" presencia de proyectos y trabajos realizados a la fecha por las instituciones 
que participan en alguna medida en la conformación de la Infraestructura Colombiana de Datos Espaciales, y que vayan encaminados específicamente al municipio de Chinácota, su entorno o la región.

Seguros de esto, hemos querido presentar una propuesta elemental de modo que sirva de referencia básica para cuando llegue el momento de la implementación y puesta en marcha del primer geoportal de la Infraestructura de Datos Espaciales del Municipio de Chinácota (Geoportal IDE Chinácota), y de la región.

\section{Referencias}

[1] MOYA-HONDUVILLA, Javier. "Análisis y Diseño de Alternativa al Geoportal de la Infraestructura de Datos Espaciales de España (IDEE). Aplicación de la Metodología de Diseño Orientada a Metas (DOM) de Cooper". [Proyecto Fin de Carrera]. Director: RODRIGUEZ PASCUAL, Antonio F. Universidad Politécnica de Madrid. Escuela Superior de Ingenieros en Topografía, Geodesia y Cartografía. Madrid, Febrero 2007. A01, Pág. 6.

[2] SMITH, Michael John de, GOODCHILD, Michael F., LONGLEY, Paul. Geospatial Analysis: A Comprehensive Guide to Principles, Techniques and Software Tools. Leicester UK: Published by Matador, 2007. Pág. 50.

[3] ZAPATA JARAMILLO, Carlos Mario, GONZÁLEZ CALDERÓN, Guillermo, MARÍN MORALES, María Isabel. "Un caso de Estudio en Interoperabilidad entre Sistemas de Información Geográfica”. En: Sistema de Información Científica, Red de Revistas Científicas de América Latina, el Caribe, España y Portugal. Redalyc, Revista Ingenierías Universidad de Medellín, Colombia, Vol. 8, núm. 15, julio-diciembre, 2009. Pág.
11-20 Disponible en [http://redalyc. uaemex.mx/src/inicio/ArtPdfRed. jsp?iCve $=75017199003]$.

[4] KRESSE, Wolfgang; DANKO, David. "Cap. 12. Geospatial Metadata". En: Handbook of Geographic Information. New York: Editorial Springer, 2012. Pág. 362.

[5] TSAO SANTÍN, Francisco Javier. "Introducción a los servicios OpenGIS". En: IX Jornadas sobre Software Libre. Grupo de Programadores y Usuarios de Linux - Grupo de Ingeniería Cartográfica de la Escuela de Ingenieros de Caminos, Canales y Puertos, 21 de Abril de 2009.

[6] KRESSE, Wolfgang, DANKO, David, FADAIE, Kian. "Cap. 13. Standardization". En: Handbook of Geographic Information. New York: Editorial Springer, 2012. Pág. 394.

[7] DOMÉNECH QUESADA, Juan Luis, y SANZ LARRUGA, Francisco Javier. Guía para la Implementación de un Sistema de Gestión Integrada de Zonas Costeras. España: Netbiblo, S.L., 2010. Pág. 120.

[8] GÓMEZ DELGADO, Montserrat y CANO BARREDO, José Ignacio. Sistemas de Información Geográfica y evaluación multicriterio en la ordenación del território. Segunda edición,. Madrid: Editorial Ra-Ma, 2005. Pág. 21.

[9] Bañares, J., BERNABÉ, M., GOULD, M. et al. Aspectos Tecnológicos de la Creación de una Infraestructura Nacional Española de Información Geográfica. 2001. Disponible en [http://redgeomatica. rediris.es/metadatos/publica/articulo03.htm] [Consultado en: Junio 23, 2012]
Enero-Junio 2013 ISSN 0122-820X 
No. 1

[10] WENG, Qihao. Remote sensing and GIS Integration: theories, methods, and applications. New York: McGraw-Hill, 2010.

[11] USÓN MURILlO, Asunción, BOIXADERA LLOBET, Jaume, BOSCH SERRA, Ángela y MARTÍN, Alberto Enrique. Tecnología de Suelos: Estudio de Casos. Universidad de Lleida. Zaragoza: Edicions de la Universitat de Lleida, 2010. Pg. 163.

[12] IGN, España, Instituto Geográfico Nacional de España. "Introducción a las Infraestructuras de Datos Espaciales (IDE)". 2009. Pág.7. Disponible en [http://www.ign.es/ign/resources/ cartografiaEnsenanza/ideeEso/I-IDE/ I-IDE/recursos/I-IDE.pdf] [Consultado en: Julio 4, 2012].

[13] Secretaria Distrital de Planeación de Bogotá. "Abc del Plan de Ordenamiento Territorial de Bogotá. Nociones básicas y elementos para su revisión". 2009. Pág. 1-41. Disponible en [http:// www.sdp.gov.co/portal/page/portal/PortalSDP/POT/QueEs/abc_del_ POT (FinaloEDsimo-Feb-23-09) ERG. pdf].

[14] AGER INGENIEROS. "Los Sistema de Información Geográfica, características y aplicaciones generales". Ingeniería, Arquitectura Consultoría. 2012. Pág. 2-4. Disponible en [http://www.ager. es/productos/gis/sig.pdf] [Consultado en: Julio 13, 2012].

[15] IDEE, Infraestructura de Datos Espaciales España. "Mundo IDE". 2012. [En línea] [http://www.idee.es/Web/guest/ introduccion-a-las-ide] [Consultado en: Octubre 15, 2012].

[16] MOYA-HONDUVILLA, Javier. "Análisis y Diseño de Alternativa al Geoportal de la Infraestructura de Datos Espaciales de España (IDEE). Aplicación de la Metodología de Diseño Orientada a Metas (DOM) de Cooper". [Proyecto Fin de Carrera]. Director: RODRIGUEZ PASCUAL, Antonio F. Universidad Politécnica de Madrid. Escuela Superior de Ingenieros en Topografía, Geodesia y Cartografía. Madrid, Febrero 2007. A01, Pág. 8.

[17] GRANELL, C., GOULD, M., MANSO, M., BERNABE, M. "Spatial Data Infrastructures”. En: KARIMI, H. (ed.), Handbook of Research on Geoinformatics. Hershey: Information Science Reference. 2007.

[18] VALLEJO, Paulino. "Listado de GeoPortales IDE”. 2013. Disponible en [http://paulinovallejo.wordpress. com/2013/04/09/listageoportales-ide/] [consultado en: abril 23, 2013]

[19] FLORCZYK, Aneta Jadwiga. "Search Improvement within the Geospatial Web in the context of Spatial Data Infrastructures". Director: ZARAZAGA SORIA, Francisco Javier [Tesis doctoral] Universidad de Zaragoza. Departamento de Ciencias de la Computación, 2012.

[20] MÁRQUEZ SOLÍS, Santiago. La Web Semántica: Web Semántica y Servicios Web Semánticos. Primera Edición. 2007. Pág. 50.

[21] CROMPVOETS, J., BREGT, A., RAJABIFARD, A., and WILLIAMSON, I. "Assesing the worldwide developments of national spatial data clearinghouses". En: International Journal Geographical Information Science, 2004. Vol.18. Pág. 25.

[22] MOYA HONDUVILLA, J., BERNABÉ POVEDA, M.A., MANRIQUE SANCHO, M.T. "La Usabilidad de los Geoportales: Aplicación del Diseño Orientado a Metas (DOM)". Grupo de Investigación MERCATOR - Universidad Politécnica de Madrid. ETSI en To- 
Cúcuta-Colombia

pografía, Geodesia y Cartografía. 2007.

[23] IGN, España, Instituto Geográfico Nacional de España. "Infraestructuras de Datos Espaciales, Definición y Componentes". En: Temario y documentación de cursos. Sistemas y Tecnologías de la Información. 2008. Pág. 307. Disponible en [http://www.ign.es/ign/resources/acercaDe/aig/B.pdf] [Consultado en: Julio 21, 2012]. 